\title{
Optimized hierarchical rule-based classification for differentiating shallow and deep- seated landslide using high-resolution LiDAR data
}

\begin{abstract}
Landslide is one of the most devastating natural disasters across the world with serious negative impact on its inhabitants and the environs. Landslide is considered as a type of soil erosion which could be shallow, deep-seated, cut slope, bare soil, and so on. Distinguishing between these types of soil erosions in dense vegetation terrain like Cameron Highlands Malaysia is still a challenging issue. Thus, it is difficult to differentiate between these erosion types using traditional techniques in locations with dense vegetation. Light detection and ranging (LiDAR) can detect variations in terrain and provide detailed topographic information on locations behind dense vegetation. This paper presents a hierarchical rulebased classification to obtain accurate map of landslide types. The performance of the hierarchical rule set classification using LiDAR data, orthophoto, texture, and geometric features for distinguishing between the classes would be evaluated. Fuzzy logic supervised approach (FbSP) was employed to optimize the segmentation parameters such as scale, shape, and compactness. Consequently, a correlation-based feature selection technique was used to select relevant features to develop the rule sets. In addition, in other to differentiate between deep-seated cover under shadow and normal shadow, the band ration was created by dividing the intensity over the green band. The overall accuracy and the kappa coefficient of the hierarchal rule set classification were found to be 90.41 and $0.86 \%$, respectively, for site A. More so, the hierarchal rule sets were evaluated using another site named site B, and the overall accuracy and the kappa coefficient were found to be 87.33 and $0.81 \%$, respectively. Based on these results, it is demonstrated that the proposed methodology is highly effective in improving the classification accuracy. The LiDAR DEM data, visible bands, texture, and geometric features considerably influence the accuracy of differentiating between landslide types such as shallow and deep-seated and soil erosion types like cut slope and bare soil. Therefore, this study revealed that the proposed method is efficient and well-organized for differentiating among landslide and other soil erosion types in tropical forested areas.
\end{abstract}

Keyword: Landslide; GIS; Fuzzy logic; LiDAR; Remote sensing 